\title{
Experiments on Minority Carrier Diffusion in Silicon: Contributions of Excitons
}

\author{
J. Mohrhof* and D. Silber** \\ *REpower Systems AG, Hollesenstr. 15, 24768 Rendsburg, Germany \\ j.mohrhof@repower.de \\ ** Institute for Electrical Drives, Power Electronics and Devices, \\ University of Bremen, PO-Box 330440, 28334 Bremen, Germany \\ d.silber@et.uni-bremen.de
}

\begin{abstract}
Experiments on minority carrier diffusion using an a.c. photocurrent method exhibit very good agreement with Klaassens's model [1] at temperatures $>250 \mathrm{~K}-500 \mathrm{~K}$, but very large deviations at $100 \mathrm{~K}$ and intermediate doping levels. They can be perfectly explained using published results on exciton diffusion constants and on concentrations of excitons in electron-hole-plasma. Our results confirm strongly that simulation of bipolar devices at low temperatures requires consideration of exciton formation.
\end{abstract}

\section{Introduction}

Concentrations of excitons in the electron-hole-plasma of bipolar power devices had been determined experimentally more than 30 years ago [2]. Their contributions in carrier transport have been discussed [3, 4], but experiments on minority carrier diffusion including exciton contributions, especially at low temperatures $(\sim 100 \mathrm{~K})$ had not been performed until now.

There is also considerable discussion on Coulomb scattering effects in minority carrier mobility due to majority carrier flow $[4,5,6]$, in Haynes-Shockley type experiments and in electron-hole plasma current transport, both effects must be considered. For minority carrier diffusion, matrix transport equations are not required, but in case of considerable exciton concentrations, a three-particle transport model is necessary [3]. Our experiments were motivated to determine the minority carrier diffusion constants in a great variety of doping concentration and temperatures.

\section{Experimental Method}

We have measured the a.c. photocurrent phase shift and amplitude variation as a function of modulation frequency. The method is rather unsensitive to carrier lifetime effects as long as the modulation frequency $f_{m}$ and carrier lifetime $\tau$ fulfill the condition

$2 \pi f_{m} \gg 1 / \tau$.

Moreover, in principle $\tau$ could be eleminated using both amplitude and phase shift variations. In our evaluations, however, we used this to eleminate the surface recombination velocity influence. A further important problem was the elimination of probe internal $\mathrm{RC}$ transmission line effects (see figure 1, below). 


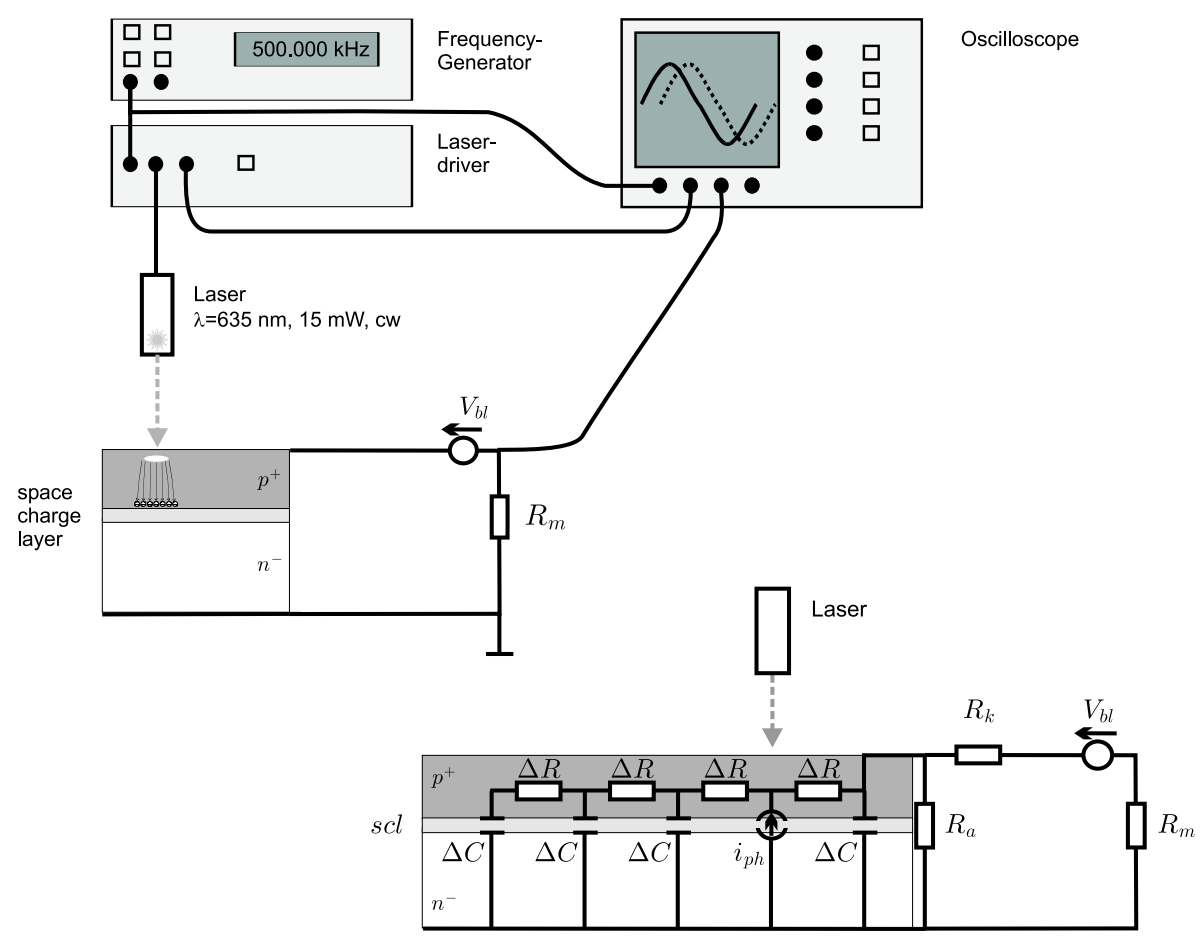

Figure 1: Experimental Setup and Probe Internal Transmission Line Effects (below)

\section{Results and Discussion}

1. Our results are in very good agreement with Klaassen's model at temperatures $250-500 \mathrm{~K}$ at nearly all doping concentrations.

2. At $100 \mathrm{~K}$, we obtained much larger effective diffusion constants at doping levels $\sim 6 \cdot 10^{16} \mathrm{~cm}^{-3}$. Considering the exciton diffusion constant measured in $[7,8]$, the exciton concentration in plasma measured in [9], and carrier freeze out effects, we obtain surprisingly exact values for the combined diffusion constant of excitons and free minority carriers (calculated line in Figures 2 and 3).

3. At high doping concentrations, it is expected that exciton formation is not possible (Mott transition) [3, 4]. The rather good agreement with Klaassen's model at high concentrations is consistent with the dissapearence of excitons above Mott transition. This indicates, that Klaassen's model is obviously correct for diffusion and drift transport of free minority carriers, but the additional contribution of excitons in diffusion can be very high.

4. Obviously Schlangenotto's results [2] on the exciton concentration in electron- 
hole-plasma (see figure 4) are well verified. This, however, has severe consequences for the interpretation of high stored charge in bipolar devices at low temperatures and on the transport of carriers. For example at $100 \mathrm{~K}$ in an electronhole-plasma of $10^{17} \mathrm{~cm}^{-3}$ concentration, $70 \%$ of carriers are "excitonic" and thus contribute to stored charge, but not to plasma conductivity.

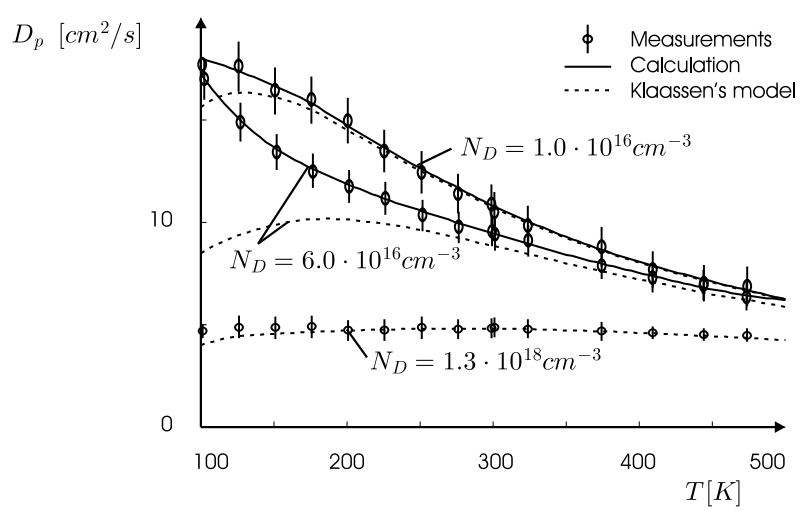

Figure 2: Temperature dependency of diffusion constants of holes in silicon $N_{D}=1.3 \cdot 10^{18} \mathrm{~cm}^{-3}$ is above of the Mott transition.

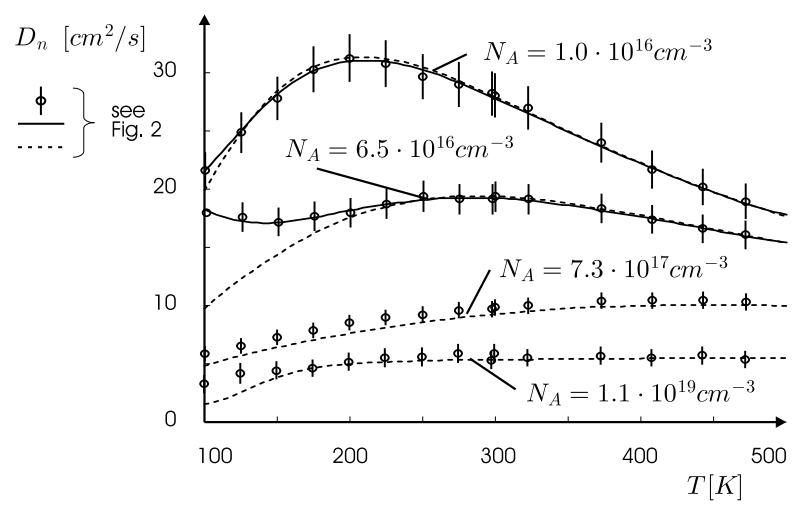

Figure 3: Temperature dependency of diffusion constants of electrons in silicon $N_{A}=7.3 \cdot 10^{17} \mathrm{~cm}^{-3}$ and $N_{A}=1.1 \cdot 10^{19} \mathrm{~cm}^{-3}$ are above of the Mott transition.

\section{Conclusion}

Obviously, exciton formation is a fact which has to be included for simulation of bipolar power devices at low temperatures. This contribution to stored charge has to be considered in the analysis of bipolar power device switching properties. More experimental and theoretical work is required to investigate the combined influence of coulomb scattering (Matrix transport) and excitons. 


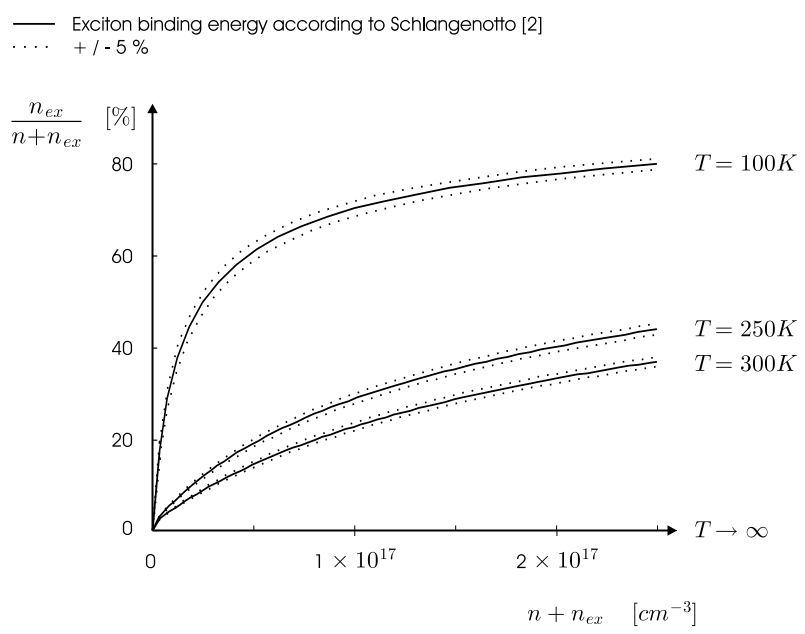

Figure 4: Relative Exciton concentration vs. total carrier concentration in the electronhole plasma according to [2]

\section{References}

[1] D.B.M. Klaassen. A unified mobility model for device simulation-I+II. Model equations and concentration dependence / Temperature dependence of carrier mobility and lifetime. Solid-State Electronics, 35(7):953-967, 1992.

[2] H. Schlangenotto, H. Maeder, and W. Gerlach. Temperature Dependence of the Radiative Recombination Coefficient in Silicon. Physica Status Solidi (a), 21:357-367, 1974.

[3] R. Corkish, D.S.-P. Chan, and M.A. Green. Excitons in silicon diodes and solar cells: A three-particle theory. Journal of Applied Physics, 79(1):195-203, January 1996.

[4] D.E. Kane and R.M. Swanson. Effect of electron-hole scattering on the current flow in semiconductors. Journal of Applied Physics, 72(11):5294-5304, December 1992.

[5] T.P. McLean and E.G.S. Paige. A Theory of the Effects of Carrier-Carrier Scattering on Mobility in Semiconductors. Journal of Physics and Chemistry of Solids, 16:220-236, 1960.

[6] T.T. Mnatsakanov. Transport Coefficients and Einstein Relation in a High Density Plasma of Solids. Physica Status Solidi (b), 143:225-234, 1987.

[7] B. Laurich, H. Hillmer, and A. Forchel. Optical time-of-flight investigation of the exciton transport in silicon. Journal of Applied Physics, 61(4):1480-1485, February 1987.

[8] M.A. Tamor and J.P. Wolfe. Drift and Diffusion of Free Excitons in Si. Physical Review Letters, 44(25):1703-1706, June 1980.

[9] W. Gerlach, H. Schlangenotto, and H. Maeder. On the Radiative Recombination Rate in Silicon. Physica Status Solidi (a), 13:277-283, 1972.

This work was supported by the Deutsche Forschungsgemeinschaft (DFG). 